\title{
Explaining the Higgs decays at the LHC with an extended electroweak model
}

\author{
Alexandre Alves $^{1}$, E. Ramirez Barreto ${ }^{2, a}$, A.G. Dias ${ }^{2}$, C.A. de S. Pires ${ }^{3}$, Farinaldo S. Queiroz ${ }^{3,4}$, \\ P.S. Rodrigues da Silva ${ }^{3}$ \\ ${ }^{1}$ Departamento de Ciências Exatas e da Terra, Universidade Federal de São Paulo, Diadema, SP, 09972-270, Brazil \\ ${ }^{2}$ Centro de Ciências Naturais e Humanas, Universidade Federal do ABC, Santo André, SP, 09210-170, Brazil \\ ${ }^{3}$ Departamento de Física, Universidade Federal da Paraíba, Caixa Postal 5008, João Pessoa, PB, 58051-970, Brazil \\ ${ }^{4}$ Center for Particle Astrophysics, Fermi National Accelerator Laboratory, Batavia, IL 60510, USA
}

Received: 23 November 2012 / Revised: 9 January 2013 / Published online: 1 February 2013

(c) Springer-Verlag Berlin Heidelberg and Società Italiana di Fisica 2013

\begin{abstract}
We show that the observed enhancement in the diphoton decays of the recently discovered new boson at the LHC, which we assume to be a Higgs boson, can be naturally explained by a new doublet of charged vector bosons from extended electroweak models with $\mathrm{SU}(3)_{C} \otimes$ $\mathrm{SU}(3)_{L} \otimes \mathrm{U}(1)_{X}$ symmetry. These models are also rather economical in explaining the measured signal strengths, within the current experimental errors, demanding fewer assumptions and less parameters tuning. Our results show a good agreement between the theoretical expected sensitivity to a 126-125 GeV Higgs boson, and the experimental significance observed in the diphoton channel at the $8 \mathrm{TeV}$ LHC. Effects of an invisible decay channel for the Higgs boson are also taken into account, in order to anticipate a possible confirmation of deficits in the branching ratios into $Z Z^{*}, W W^{*}$, bottom quarks, and tau leptons.
\end{abstract}

\section{Introduction}

It was announced recently at the CERN Large Hadron Collider (LHC) the discovery of a new boson whose observed properties until now suggest it is the Standard Model (SM) Higgs boson [1, 2]. Denoting by $h$ such new boson, its observation was based on decay signals in four leptons, $h \rightarrow l^{+} l^{-} l^{+} l^{-}$, and diphotons, $h \rightarrow \gamma \gamma$, with both pointing to an invariant mass of $m_{h}=125-126 \mathrm{GeV}$. The diphoton channel points to an excess over what is expected from the SM. We shall consider in this work that the $h$ is indeed a Higgs boson, i.e., as resulting from spontaneous symmetry breaking [3-5].

\footnotetext{
a e-mail: elmerraba@gmail.com
}

Within the observed mass range, several decay channels for the SM Higgs boson are experimentally accessible making possible the measurement of its coupling strength to many particles. It is not clear yet from the present data if $h$ has its couplings to fermions as dictated by the SM, even adding the latest results from Tevatron [6] which indicate an excess of $b$-jets events, probably due to the decay $h \rightarrow b \bar{b}$. We expect that this will soon be resolved with more accumulated data. If the tendency of smaller branching ratios of $h$ into $b$ quarks and $\tau$ leptons is confirmed, this could be a smoking gun for models with a fermiophobic Higgs or models with a decreased effective coupling of the Higgs with the gluons. On the other hand, if these couplings have the strength as in the SM but their branching ratios turn out to be smaller than the expected, then invisible decay channels may have an important role.

In a previous work [7] we investigated in what extension an excess for the diphoton channel can be used to probe new vector bosons within a specific framework of a class of $\mathrm{SU}(3)_{C} \otimes \mathrm{SU}(3)_{L} \otimes \mathrm{U}(1)_{X}$ gauge models, minimal 331 model for short [8-10].

Our updated results show good agreement between our theoretical expected sensitivity to a 126-125 GeV Higgs boson and the experimental significance observed in the diphoton channel at the $8 \mathrm{TeV}$ LHC.

Facing the new experimental reality we now present a new focus in the diphoton channel, showing how the observed excess of photons can be explained by a new $\mathrm{SU}(2)_{L}$ doublet of vector bosons. Such a doublet is contained in the minimal 331 model but may be part of other models with an extended electroweak gauge sector as well.

We also have included an analysis of an invisible decay width for the Higgs boson, in order to anticipate a possible confirmation of deficits in the branching ratios into $Z, W$, 
bottom quarks, and tau leptons. This class of models is truly the most economical one in the sense that all the tree level couplings of the Higgs boson to the $Z, W$, and fermions can be made exactly the same as the SM ones at the same time its possible to enhance the effective 1-loop coupling to photons. Moreover, there are not new contributions to the effective coupling between the Higgs and the gluons, thus all the Higgs production rates coincides with the SM predictions.

The quest for a mechanism of enhancement in the diphoton channel, in accordance with the recent results of [1,2], was treated in [11-20] taking into account specific models. Several independent analyses indicate deviations from the SM expectations [21, 22], and also include an invisible branching decay rate [23-26] in order to explain the discrepancies.

We found that our results are consistent with these works. Our analysis shows that a new $\mathrm{SU}(2)_{L}$ doublet of charged vector bosons of masses $\approx 213 \mathrm{GeV}$ and an $\mathcal{O}(10 \%)$ branching ratio of the Higgs boson into invisible states can reasonably fit part of the available data released by the LHC and Tevatron collaborations on Higgs branching ratios.

\section{Higgs-vector bosons interactions}

The doublet of vector bosons we take into account here has hypercharge $Y=3$

$\mathcal{V}=\left(\begin{array}{c}U^{++} \\ V^{+}\end{array}\right) \sim(2,3)$.

Let $m_{W}$ be the $W$ boson mass, and the adopted equality $m_{U}=m_{V}$ for the masses of these new vector bosons, $U$ and $V$. Such mass equality, in fact, is a good approximation once the exact mass relation furnishes $\left|m_{U}^{2}-m_{V}^{2}\right|<m_{W}^{2}$. A small shift in the charged vector masses is needed to evade constraints from the electroweak precision data as we discuss in Sect. 4, but a small degeneracy breaking does not affect our forthcoming results, so we keep the $U$ and $V$ masses equal in order to reduce the number of model parameters to a single mass parameter plus a branching ratio into invisible states, as discussed in the next section.

As the new vector bosons masses are related with an energy scale $v_{\chi}$, above the electroweak scale $v_{W}=246 \mathrm{GeV}$, their contribution to the process amplitude is multiplied by a suppression factor $m_{W}^{2} / m_{V}^{2}$ in comparison with that one coming from the $W$ boson. But there is still a significant increasing of the branching $B R(h \rightarrow \gamma \gamma)$ so that a signal above the SM is indeed observed for an interesting range of $m_{V}$. This is due a dominant contribution of vector gauge bosons, and the fact that a double charged one leads to a factor four multiplying the suppression factor.

Interactions of the Higgs boson field with the new vector bosons are described by the following interaction Lagrangian:

$$
\begin{aligned}
\mathcal{L}_{H V V}= & 2\left(\sqrt{2} G_{F}\right)^{\frac{1}{2}} m_{W}^{2} h\left(c_{U} U^{++\mu} U_{\mu}^{--}+c_{V} V^{+\mu} V_{\mu}^{-}\right) \\
& +g^{2} h^{2}\left(U^{++\mu} U_{\mu}^{--}+V^{+\mu} V_{\mu}^{-}\right)
\end{aligned}
$$

where $G_{F}$ is the Fermi constant, with $c_{U}$ and $c_{V}$ coefficients of order one. We take here the specific configuration of the models in Ref. [7] where $c_{U}=c_{V}=\frac{1}{2}$. This choice is made in order to simplify our analysis and have the couplings of $h$ with the bottom quark, top quark and the $W$ boson, as equal to the SM Higgs boson. We achieve this by taking two vacuum expectation values equal and additional conditions as in Appendix of Ref. [7]. ${ }^{1}$ It still has to be said that the above choice does not represent a fine tuning. There are many other allowed values for $c_{U}$ and $c_{V}$, which are functions of vacuum expectation values and some scalar fields self-couplings, producing the same essential effect of giving significant contribution of $U$ and $V$ in the diphoton Higgs boson decay.

Also, we checked that contributions due the couplings of the Higgs boson with additional charged scalars are small enough for being disregarded. In fact, a charged scalar with mass comparable with the vector bosons $U$ and $V$ can only give sub-dominant contributions.

The diphoton Higgs boson decay is described by the effective Lagrangian

$\mathcal{L}_{H \gamma \gamma}=\frac{\alpha\left(\sqrt{2} G_{F}\right)^{\frac{1}{2}}}{8 \pi}\left(F^{\mathrm{SM}}+F^{\mathrm{new}}\right) h F^{\mu \nu} F_{\mu \nu}$.

$\alpha$ is the fine structure constant, $F^{\mathrm{SM}}$ and $F^{\text {new }}$ are structure function coefficients. $F^{\mathrm{SM}}$ is what is obtained taking into account the interactions of the SM Higgs boson. The expression for $F^{\mathrm{SM}}$ can be found in Refs. [27-30]. All nonstandard couplings of the Higgs boson field with electrically charged fields gives rise to $F^{\text {new }}$. In the case we are considering, this last coefficient is obtained from the trilinear interaction in Eq. (2) and new vector boson coupling with the photon. The Feynman diagrams involved are shown in Fig. 1. The result of these diagrams can be obtained from the corresponding ones for $W$ boson [27-30] just multiplying by a scale factor

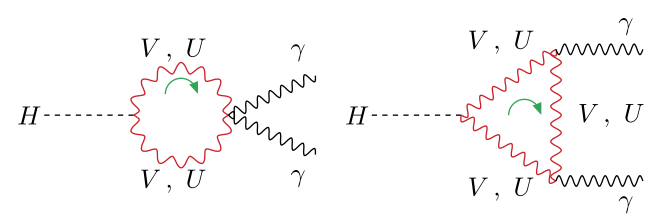

Fig. 1 The one-loop diagrams involving the new charged gauge bosons $U$ and $V$ which contribute most to the $h \rightarrow \gamma \gamma$ decay amplitude

\footnotetext{
${ }^{1}$ We take here the condition on the scalar fields self-couplings $\lambda_{1}=\lambda_{2}$ in the appendix of Ref. [7]. Such a simplification also leads to a maximal mixing between $h=h_{1}$ and another scalar $h_{2}$ which, naturally, could be heavier than $h$.
} 
proportional to $c_{U} m_{W}^{2} / m_{V}^{2}$. Thus, we have

$F^{\text {new }}=5\left[2+3 \tau_{V}+3 \tau_{V}\left(2-\tau_{V}\right) I^{2}\right] \frac{m_{W}^{2}}{2 m_{V}^{2}}$,

with

$\tau_{V} \equiv \frac{4 m_{V}^{2}}{m_{h}^{2}}$,

and

$I \equiv \begin{cases}\arcsin \left(\sqrt{\frac{1}{\tau_{V}}}\right) & \text { for } \tau_{V} \geq 1, \\ \frac{1}{2}\left[\pi+\imath \ln \left[\frac{1+\sqrt{1-\tau_{V}}}{1-\sqrt{1-\tau_{V}}}\right]\right] & \text { for } \tau_{V} \leq 1 .\end{cases}$

For the diphoton Higgs boson decay rate we have, therefore,

$\Gamma_{h \gamma \gamma}=\frac{\alpha^{2} m_{h}^{3} G_{F}}{128 \sqrt{2} \pi^{3}}\left|F^{\mathrm{SM}}+F^{\text {new }}\right|^{2}$.

\section{Observing a Higgs boson from 331 models with and without dark matter}

In 331 models, the light Higgs boson couples to the $\mathrm{SU}(2)_{L}$ doublets of new gauge bosons and scalars but not to the new fermions, so we assume that the cross sections for the main light Higgs production processes are the same as the SM at the LHC and the Tevatron, i.e.

$\sigma_{331}(g g \rightarrow h)=\sigma_{s m}(g g \rightarrow h)$,

$\sigma_{331}^{v b f}(p p(\bar{p}) \rightarrow h j j)=\sigma_{s m}^{v b f}(p p(\bar{p}) \rightarrow h j j)$,

$\sigma_{331}(p p(\bar{p}) \rightarrow h Z(W))=\sigma_{s m}(p p(\bar{p}) \rightarrow h Z(W))$.

The new charged gauge bosons mediate interactions between exotic and SM quarks only, which is why the 1-loop effective gluon-gluon-Higgs coupling does not receive new contributions. All the tree level couplings between the Higgs and all the fermions, the $Z$, and the $W$ bosons, are the same as the SM. On the other hand, the effective 1-loop coupling to $\gamma \gamma$ and $Z \gamma$ receive contributions from the new charged gauge bosons, $V^{ \pm}$and $U^{ \pm \pm}$, and the charged scalars. With no other particles to decay to, the experimental signatures expected for the Higgs boson in 331 models should look very similar to the SM, but the channels related to photons decays.

As we pointed out in the previous section, the impact of the charged scalars on the branching ratio of the Higgs boson in two photons $B R_{331}(h \rightarrow \gamma \gamma)$ is negligible. We checked that for $m_{V} \gtrsim 100 \mathrm{GeV}$, the contribution from scalars amount to less than $2 \%$ for $100 \mathrm{GeV}$ charged vectors. As we will see, the preferred charged vector masses that fit the available data lie in the region $m_{V}>150 \mathrm{GeV}$, so we can safely neglect the charged scalars in the calculations.

We are going to show in this section that a model with an extended gauge sector, as the 331 models, is able to explain the current observed Higgs branching ratios at the LHC within the current statistical errors. We also emphasize that there exist minimal 331 constructions that possess a cold dark matter (CDM) candidate. This is a key feature for and experimentally well founded new physics model.

Although the minimal 331 model, as presented in Ref. [7], does not contain a CDM candidate, it can be embedded in a larger gauge group, 341 [31] at least, which has a neutral scalar which can be the lightest typical 341 particle, ${ }^{2}$ and its mass may be varied such as to lead to a suitable CDM candidate [32].

Even more interesting, when the minimal 331 is supersymmetrized [33, 34] (SUSY331), the lightest supersymmetric particle, generally a neutralino, is a good CDM candidate protected by R-parity, as in the Minimal Supersymmetric Standard Model (MSSM) [35]. Considering the observation of an $125 \mathrm{GeV}$ Higgs boson, however, the supersymmetric 331 presents an important advantage over the MSSM - the upper bound on the Higgs boson mass lies comfortably above the measured mass when radiative corrections are considered [33, 34, 36], demanding less fine tuning (or none) in the parameters of the model, mainly the scalar top mass.

In both cases just discussed, it is reasonable to keep only the lightest particle, the CDM candidate, at low energy scale (some 10 to $100 \mathrm{GeV}$ ), while the remaining extra fields in the spectrum may be at the $\mathrm{TeV}$ scale or so, thus decoupling from the electroweak breaking regime and playing no role in the Higgs decay branching ratios. Moreover, we will show that if the branching ratios of the Higgs into the other SM particles are smaller than the predicted by the SM, then the invisible decay mode can be a natural way to decrease the branching fractions [26]. From now, on whenever we speak of the minimal 331 model we mean a 331 model with no DM candidate, while 331DM will refer to those models with a DM candidate (341 or SUSY331).

We define the ratio $\mu_{\gamma \gamma}$ between the branching fraction of a Higgs boson decaying into two photons of the 331 models and the SM, as follows:

$\mu_{\gamma \gamma}=\frac{B R_{331}(h \rightarrow \gamma \gamma)}{B R_{s m}(h \rightarrow \gamma \gamma)}$

and between the $s=Z, W, b, \tau$ branching ratios from the 331 and the Standard Model

$\mu_{s s}=\frac{B R_{331}(h \rightarrow s s)}{B R_{s m}(h \rightarrow s s)}$.

The SM Higgs boson widths and branching ratios were computed with the HDECAY [37] program.

\footnotetext{
${ }^{2}$ This particle can be made stable by imposing a symmetry that transforms only the 341 fields which are singlet under the 331 symmetry. It is in this sense that we call it a typical 341 particle.
} 


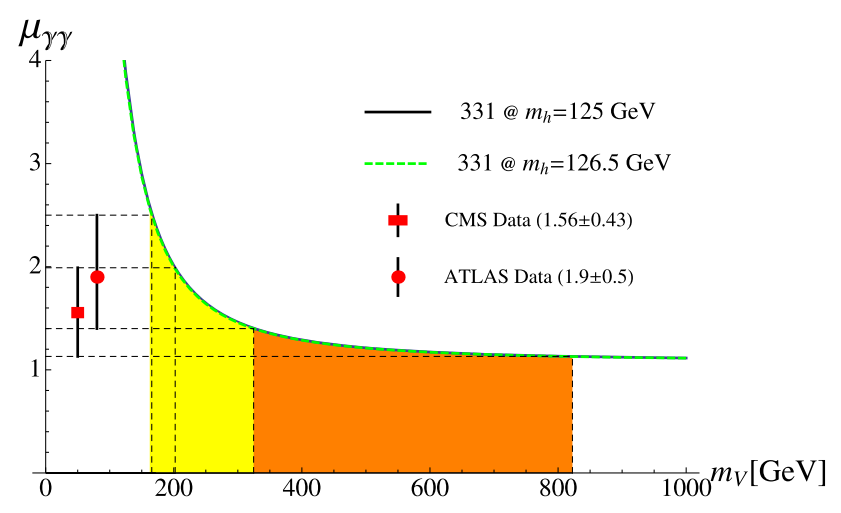

Fig. 2 The ratio $\mu_{\gamma \gamma}$ as a function of the common charged vector bosons masses $m_{V}$ for $m_{h}=126.5 \mathrm{GeV}$ (solid line) and $m_{h}=125 \mathrm{GeV}$ (dashed green line). We also show the $\mu_{\gamma \gamma}$ constraint from the latest CMS and ATLAS data points on the charged vector masses. The two lines are hardly distinguishable in this scale

We show $\mu_{\gamma \gamma}$ as a function of the $m_{V}$ masses in Fig. 2. The solid line represents $\mu_{\gamma \gamma}$ from a minimal 331 model with $m_{h}=125 \mathrm{GeV}$, the central value from CMS, and the dashed line a $m_{h}=126.5 \mathrm{GeV}$ Higgs, the preferred value from ATLAS (almost indistinguishable from the solid line). In order to illustrate the experimental constraint from the recent LHC data on the Higgs search, we plot the $\mu_{\gamma \gamma}^{\mathrm{CMS}}=$ $(1.56 \pm 0.43)$ and $\mu_{\gamma \gamma}^{\text {ATLAS }}=(1.9 \pm 0.5)$ data points as measured by the CMS and ATLAS [1,2] collaborations, respectively.

For the $1 \sigma$ band variation $1.13<\mu_{\gamma \gamma}^{\mathrm{CMS}}<1.99$, the $m_{V}$ masses lie in the range $(200 \mathrm{GeV}, 825 \mathrm{GeV})$, and in the range $(170 \mathrm{GeV}, 320 \mathrm{GeV})$ for $1.4<\mu_{\gamma \gamma}^{\mathrm{ATLAS}}<2.4$ as can be seen in Fig. 2. Of course, as the uncertainty in the data decreases these ranges will become narrower and a more precise prediction will be possible. Notwithstanding, these sub$\mathrm{TeV}$ mass ranges seem to be well within the search reach of the $8 \mathrm{TeV}$ LHC. Similar constraints follow from earlier ATLAS and CMS data.

In the analysis made in Ref. [7], a Higgs boson with mass $125 \mathrm{GeV}$ was found to give a $\sim 3 \sigma$ signal at the LHC, after $5 \mathrm{fb}^{-1}$ of integrated luminosity have been accumulated, for new charged gauge bosons masses of $280 \mathrm{GeV}$. In Fig. 3 we update the expected significances at the $8 \mathrm{TeV}$ LHC with 5.3 and $5.9 \mathrm{fb}^{-1}$ for CMS and ATLAS, respectively, for minimal 331 models. This picture will not change too much for 331DM models, however. ${ }^{3}$ The blue points in the figure represents the solution for the vector boson mass obtained from $m_{h}=126.5(125) \mathrm{GeV}$ and $\mu_{\gamma \gamma}=1.9(1.53)$, the experi-

\footnotetext{
${ }^{3}$ In 341 models, the expected signal rate for the process $p p \rightarrow h \rightarrow$ $\gamma \gamma$ is the same as the minimal 331. The SUSY331 gives additional contributions both to the Higgs boson production in gluon fusion, as the Higgs decay to photons. However, for a heavy SUSY spectrum the extra states would have a small impact on $\sigma \times B R(h \rightarrow \gamma \gamma)$.
}
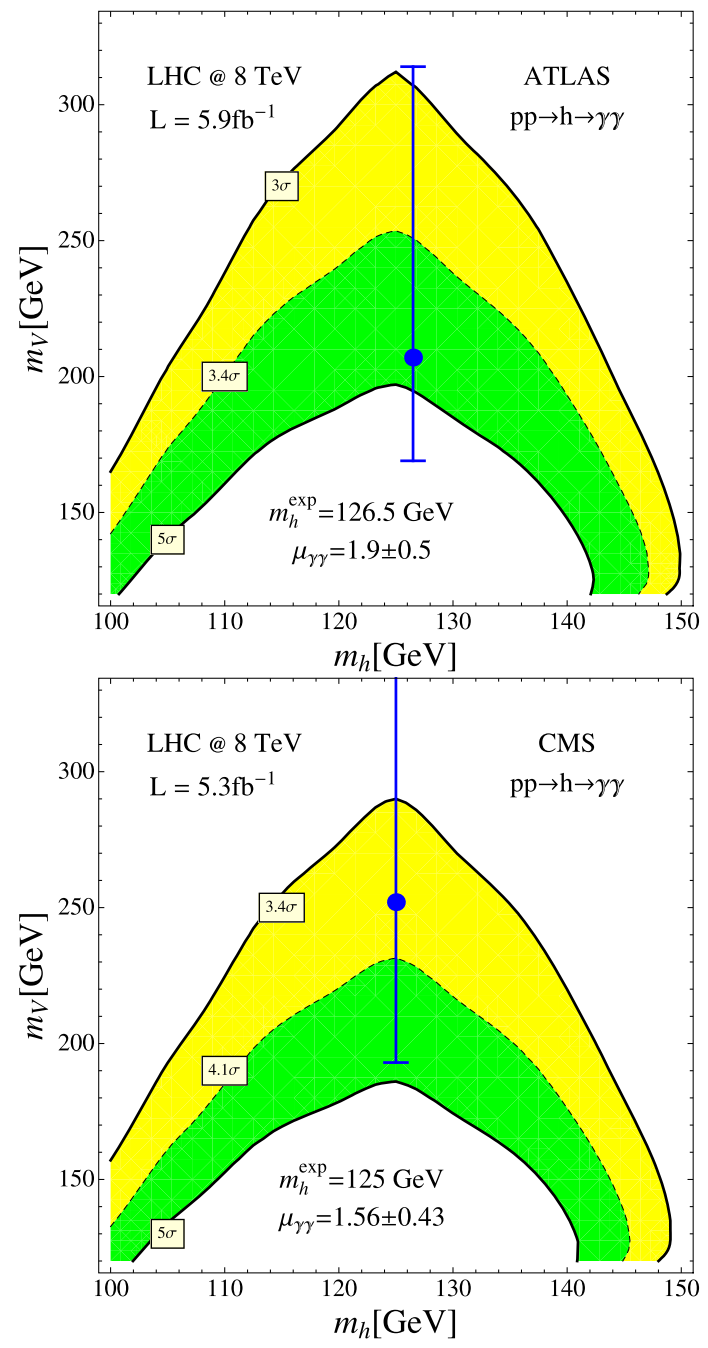

Fig. 3 In the bottom (top) panel we show the expected sensitivity of the LHC $8 \mathrm{TeV}$ after 5.3(5.9) $\mathrm{fb}^{-1}$ of collected data at the CMS (ATLAS) in the $g g \rightarrow h \rightarrow \gamma \gamma$ channel updated from Ref. [7]. The blue bars represent the mass constraint on the charged vectors from the minimal 331 model from the latest CMS (ATLAS) data on the signal strength $\mu_{\gamma \gamma}$ (Color figure online)

mental central values from CMS (ATLAS), while the blue bars represent the uncertainty in these data.

Note that expected significances are remarkably close to the observed significances of 3.4 (4.1) $\sigma$ from CMS (ATLAS) [1, 2] for the $h \rightarrow \gamma \gamma$ channel only. We point out, however, that the analysis made in Ref. [7] uses a less powerful statistics for the hypothesis test and somewhat different kinematic cuts compared to the experimental analysis. On the other hand, we calculated the main reducible and all the irreducible backgrounds at NLO accuracy, including single and double bremsstrahlung effects, and the Higgs production in gluon fusion at NLO QCD+EW. See Ref. [7] for more details.

There is a number of ways to confront the minimal 331 and 331DM model explanation to other candidate models. 
First of all, the new heavy 331 quarks do not couple to the Higgs boson as discussed in previous section. If the susy spectrum is heavy enough, the contributions from SUSY331 will also be negligible. So, the $h g g$ coupling is of SM sizemany other extensions give extra contributions to this coupling increasing the Higgs production cross section in gluon fusion. The $h \rightarrow Z \gamma$ is expected to change as well due the same new gauge bosons running in the loop. Direct search for the new gauge bosons would be the ultimate test, once their masses would be of sub-TeV order and possibly accessible to the LHC.

As we pointed out before, the tree level couplings of the Higgs boson to the $Z, W, g$ bosons and to all the fermions are identical to the SM ones in 331 models. It means that if the Higgs boson decays to new states then all branching fractions to SM particles, dominated by tree level couplings, will be affected by the same factor. A few models realize this situation in a more natural fashion, for example, a spontaneously broken $\mathcal{N}=1$ SUSY with a sgoldstino [17] is able to enhance the diphoton signal and keep the other branching ratios untouched at the cost of requiring either the wino or the bino to have a mass of the order of the gluino to avoid an overall enhancement due a larger $h g g$ coupling. Another example is a Higgs impostor, as the Randall-Sundrum radion proposed in [18], where the $h \gamma \gamma$ and $h g g$ are enhanced due to trace anomaly. In these two examples, a reduction in the branching ratios to SM particles, but the photon, can be achieved increasing the $B R(h \rightarrow g g)$. Unfortunately, it is very difficult to detect this decay channel even in the Higgsstrahlung process due the overwhelming QCD backgrounds at hadron colliders.

If the Higgs decays to a pair of dark matter particles then, denoting such branching by $\alpha=B R(h \rightarrow$ invisible $)$, the branching to a SM particle will be changed as follows

$$
\begin{aligned}
& B R_{331}(h \rightarrow s s) \\
& =\frac{\Gamma_{s s}^{\mathrm{SM}}}{\tilde{\Gamma}_{\mathrm{SM}}+\Gamma_{\gamma \gamma}^{331}\left(m_{V}\right)+\frac{\alpha}{1-\alpha}\left[\tilde{\Gamma}_{\mathrm{SM}}+\Gamma_{\gamma \gamma}^{331}\left(m_{V}\right)\right]} \\
& =\frac{(1-\alpha) \Gamma_{s s}^{\mathrm{SM}}}{\tilde{\Gamma}_{\mathrm{SM}}+\Gamma_{\gamma \gamma}^{331}\left(m_{V}\right)} .
\end{aligned}
$$

In this formula, $s$ denotes all the SM particles but the photon and $\tilde{\Gamma}_{\mathrm{SM}}=\Gamma_{\text {tot }}^{\mathrm{SM}}-\Gamma_{\gamma \gamma}^{\mathrm{SM}}$. The branching ratio to photons in 331 models is given similarly by

$$
B R_{331}(h \rightarrow \gamma \gamma)=\frac{(1-\alpha) \Gamma_{\gamma \gamma}^{331}\left(m_{V}\right)}{\tilde{\Gamma}_{\mathrm{SM}}+\Gamma_{\gamma \gamma}^{331}\left(m_{V}\right)}
$$

where $\Gamma_{\gamma \gamma}^{331}\left(m_{V}\right)$ is the partial width for a pair of photons as a function of the new gauge boson masses $m_{V}$.

The effect of an invisible decay channel in the Higgs branching ratios into SM particles is to linearly decrease these branching ratios. If we want to keep $\mu_{\gamma \gamma}$ in the ballpark of the experimental CMS value, for example, we need

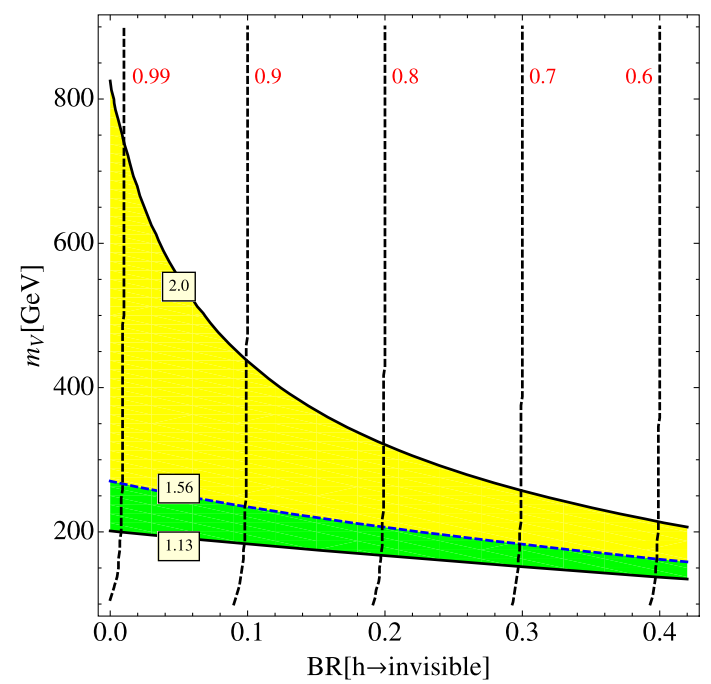

Fig. 4 The effect of an invisible decay channel in the ratio $\mu_{\gamma \gamma}$ in 331DM models. The green (yellow) band represents the downward (upward) $1 \sigma$ mass constraint from the CMS data [1, 2]: $1.13<\mu_{\gamma \gamma}^{\mathrm{CMS}}<2.0$. The dashed blue line represents the experimental central value. The almost vertical dashed lines show $\mu_{s s}$ as a function of the invisible branching ratio (Color figure online)

lighter new gauge bosons. This can be seen in Fig. 4 where we show $\mu_{\gamma \gamma}$ and $\mu_{s s}$ in the $m_{V}$ versus $B R(h \rightarrow$ invisible $)$. The blue dashed line shows the central experimental CMS value for $\mu_{\gamma \gamma}$ and the green (yellow) band the $1 \sigma$ downward (upward) variation. The dashed vertical lines show $\mu_{s s}$ in the $m_{V}$ versus $B R(h \rightarrow$ invisible $)$ plane. Their values are quite insensitive to $m_{V}$ values, but decreases linearly with $\alpha$.

\section{Confronting Higgs decays from 331 models to the LHC and Tevatron data}

Despite the current data being compatible with the SM predictions within the current experimental errors, it has been shown that some sensitivity to new phenomena might be reached already combining all the released data from LHC and Tevatron collaborations [21-25]. In fact, these works suggest that the couplings of the Higgs boson to the weak gauge bosons and fermions are compatible to the SM values, except for the photons possibly. Besides, a branching ratio into invisible states can be accommodated [23, 25]. This situation can be naturally explained within the 331 models presented here.

A closer look at the data shows that many measurements indicate a smaller signal strength compared to the SM expectations. ${ }^{4}$ In order to study the possibility of an invisible decay channel plus an enhanced branching ratio into pairs of photons within the 331 models, we performed a $\chi^{2}$ analysis

\footnotetext{
${ }^{4}$ See Ref. [22] for a good compilation of the relevant experimental data.
} 
using part of the available data from the LHC and Tevatron collaborations. For that aim we construct the following $\chi^{2}$ statistics with $m_{V}$ and $\alpha=B R(h \rightarrow$ invisible $)$ as free parameters:

$$
\begin{aligned}
\chi^{2}= & \sum_{n=1}^{N_{\exp }}\left[\sum_{s=Z, W, b, t}\left(\frac{\mu_{n, s s}^{\exp }-\mu_{s s}\left(m_{V}, \alpha\right)}{\sigma_{n, s s}^{\exp }}\right)^{2}\right. \\
& \left.+\left(\frac{\mu_{n, \gamma \gamma}^{\exp }-\mu_{\gamma \gamma}\left(m_{V}, \alpha\right)}{\sigma_{n, \gamma \gamma}^{\exp }}\right)^{2}\right]
\end{aligned}
$$

where $N_{\exp }$ ranges from 1 [38], 2 [39], 3 [1, 2], 4 [40], to $5[1,2]$. We quote these data in Table 1 . The quoted experimental errors are asymmetric but we take the average of the upper and lower variances to compute $\sigma_{s s}^{\exp }, \sigma_{\gamma \gamma}^{\exp }$, and the $\chi^{2}$ statistics. The experimental collaborations do not provide us the correlation matrices, so we can take into account neither the possible correlations among the experimental data sets of the collaborations nor between the 7 and $8 \mathrm{TeV}$ runs. We also do not take systematic uncertainties (as the theory errors on the production cross sections) into account, so our results should be taken as a rough estimate of the best $m_{V}$ masses and invisible branching ratio which fit the data therefore.

After computing the global $\chi^{2}$ we determine the minimum $\chi_{\min }^{2}$ and plot the modified statistics $\Delta \chi^{2}=\chi^{2}-\chi_{\min }^{2}$ in Fig. 5 where the $60 \%, 68 \%, 70 \%$, and $80 \%$ C.L. contours are shown in the $m_{V}$ vs. $B R(h \rightarrow$ invisible $)$ plane. The contour values, for a given confidence level $\lambda$, are calculated from the inverse cumulative distribution function (CDF)of the $\chi_{n_{\text {dof }}}^{2}$ probability density for $n_{\text {dof }}=2$ degrees of freedom: $\Delta \chi_{\lambda}^{2}=\mathrm{CDF}_{\chi^{2}}\left(1-\lambda, n_{\mathrm{dof}}\right)$. The $68 \%$ C.L. corresponds to $\Delta \chi_{0.68}^{2}=2.30$, for example [41]. From our fitting procedure we found $\chi_{\min }^{2}=13.72$ for 20 data points. The $\chi^{2}$ for the SM hypothesis is 17.23 which is inside the $84 \%$ region in the $m_{V}$ versus $B R(h \rightarrow$ invisible $)$ plane and agrees well with the number found in [22], for example.

The best fitted point in this parameters space is $\left[m_{V}\right.$, $B R(h \rightarrow$ invisible $)]=[212.5 \mathrm{GeV}, 0.17]$, and the best theoretical signal strengths corresponding to these values are $\mu_{\gamma \gamma}=1.57$ and $\mu_{s s}=0.83$ which agree reasonably well with the CMS and ATLAS values [1,2], while the $68 \%$ C.L. intervals are $(164.4 \mathrm{GeV}, 471.7 \mathrm{GeV})$ and $1.02<\mu_{\gamma \gamma}<2.1$.
We point out that a $17 \%$ branching ratio in invisible decays is in fairly good agreement with similar analysis made in Refs. [23, 25]. Moreover, a general fit of the Higgs couplings [22] found that the present data favor a $55 \%$ smaller Higgs production rate in gluon fusion compared to the SM rate. This is a consequence of the deficits found in 13 out of the 29 data points used in the fitting procedure in that work. If we suppose this is not an effect of a fainter hgg coupling, an invisible decay is the best alternative to a global decrease in the observed Higgs boson branching ratios.

The fitting is dominated by the $\gamma \gamma, Z Z^{*}$, and $W W^{*}$ data from ATLAS and CMS which quote the smaller experimental errors. Despite a somewhat large branching to invisible decays is preferred by the data in order to fit the dominating $Z Z, W W$ channels, a vanishing $B R(h \rightarrow$ invisible $)$ is within the $68 \%$ confidence interval. The SM point lies in $B R(h \rightarrow$ invisible $)=0$ line for large $m_{V}$. On the other hand, a SM branching into photons seems less favored by the current data.

Supposing that the Higgs boson decays to SM particles exclusively we fit $B R(h \rightarrow \gamma \gamma)$ to the data as a function of

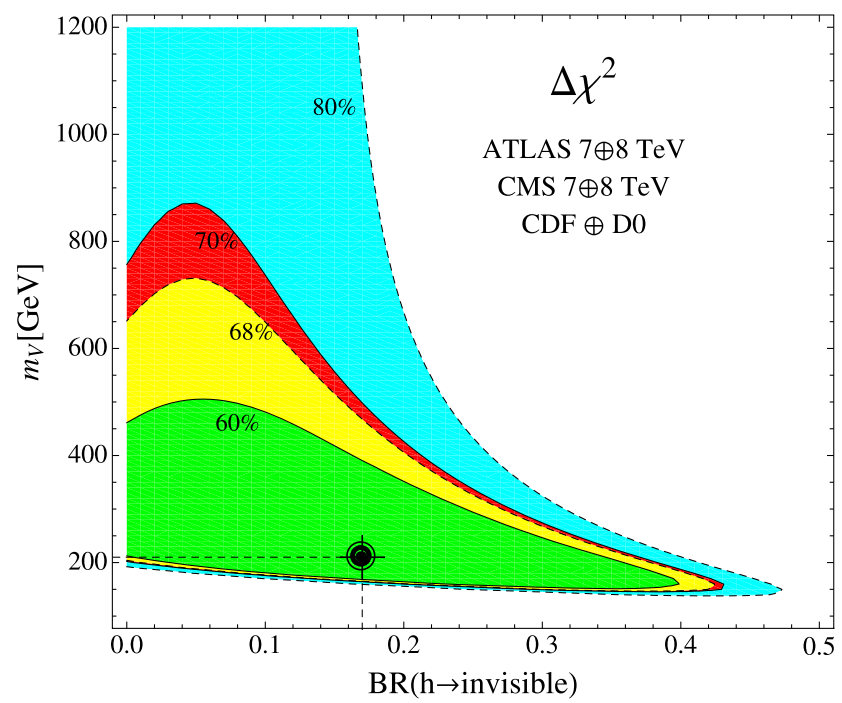

Fig. 5 The $\Delta \chi^{2}$ contours in the plane $m_{V}$ versus $\alpha=$ $B R[h \rightarrow$ invisible $]$ corresponding to the Confidence Levels of $60 \%, 68 \%, 70 \%$, and $80 \%$ computed from Table 1 . The plotted point locates the best fitted parameters from the data

Table 1 Experimental data used in the fitting procedure. The symmetric errors are computed from the actual asymmetric experimental errors by averaging their variances

\begin{tabular}{llllll}
\hline$N_{\exp }$ & $\mu_{\gamma \gamma}^{\exp }$ & $\mu_{Z Z^{*}}^{\exp }$ & $\mu_{W W^{*}}^{\exp }$ & $\mu_{b b}^{\exp }$ & $\mu_{\tau \tau}^{\exp }$ \\
\hline (1) ATLAS 7 TeV & $1.6 \pm 0.81$ & $1.4 \pm 0.80$ & $0.5 \pm 0.7$ & $0.5 \pm 2.05$ & $0.2 \pm 1.80$ \\
(2) CMS 7 TeV & $1.5 \pm 1.05$ & $0.6 \pm 0.77$ & $0.4 \pm 0.6$ & $0.2 \pm 1.96$ & $0.6 \pm 1.15$ \\
(3) CMS 7 + 8 TeV & $1.56 \pm 0.43$ & $0.7 \pm 0.44$ & $0.6 \pm 0.4$ & $-0.12 \pm 0.75$ \\
(4) ATLAS 7 + 8 TeV & $1.9 \pm 0.5$ & $1.3 \pm 0.6$ & - & - & - \\
(5) CDF and D0 & $3.6 \pm 2.76$ & - & $0.32 \pm 0.83$ & $1.97 \pm 0.71$ & - \\
\hline
\end{tabular}


$m_{V}$ obtaining $m_{V}=267.5 \mathrm{GeV}$ as the best fitted mass and $\mu_{\gamma \gamma}=1.59$ as the best signal strength value fitted from the data, for a Higgs boson mass of $126 \mathrm{GeV}$.

As anticipated in Sect. 1, a small shift in the charged vector masses is needed to evade constraints from $S, T, U$, and $\rho$ parameters. Taking into account only the vector boson contributions to the electroweak precision data [42-44] we estimate for the oblique parameters, in an approximation of quasi degenerated masses,

$$
\begin{aligned}
& S \approx-\frac{9\left(M_{V}-M_{U}\right)}{2 \pi m_{V}}, \\
& T \approx \frac{\left(M_{V}-M_{U}\right)^{2}}{4 \pi \sin ^{2} \theta_{W} m_{W}^{2}}+\frac{(\rho-1)}{\alpha}
\end{aligned}
$$

where $\sin ^{2} \theta_{W} \approx 0.23, \alpha \approx \frac{1}{128}$, and the $\rho=\frac{m_{W}}{\cos ^{2} \theta_{W} m_{Z}}$ parameter obtained from formulas in Ref. [45], with $m_{Z}$ being the mass of the model $Z$ boson. For example, choosing $M_{U}=256.3$ (199) GeV, $M_{V}=267.5(212.5) \mathrm{GeV}$, which are close to the best fitting masses found in this work, we have, in a parameter space region where $\rho=1, S \approx$ $-0.06(-0.09)$ and $T \approx 0.007(0.01)$. We have found several other solutions from masses of order of $200 \mathrm{GeV}$ up to $1 \mathrm{TeV}$ with $\rho=1$ where both $S$ and $T$ are small. This shows compatibility with the present electroweak precision data [46]. Other limits for $m_{V}$ could be obtained from muonium-antimuonium conversion [47], fermion pair production, and lepton-flavor violating processes $[48,49]$. But as shown in [50], these limits can be evaded considering an extended Higgs sector or less restrictive assumptions for the minimal 331 model. In special, these limits are not applicable to 331 model versions containing exotic leptons [10], once there is no way for those process to occur.

Finally, as our results reveal the importance of these new gauge bosons for the Higgs production, it is mandatory to search signals for $U$ and $V$ within the LHC energy regime. Many works about their production suggest clear signals due to their peculiar channels decay [51-55]. For example, the production and decay of $U$ and $V$ at the LHC would be given according to Fig. 6. Note that the pair production of $U$ and $V$ bosons receives additional contribution from the new $Z^{\prime}$ gauge boson from the extended electroweak gauge sector. Nevertheless, this $Z^{\prime}$ is not relevant for the particular Higgs channel we are dealing with here. The reconstruction of the invariant mass of the pair of leptons will reveal the $U$ mass as showed in Ref. [56]. In addition, the production of two leptons and missing energy will be also affected by $V$ [57].

Another possibility for typical process involving a new vector boson assumed here is the jets plus leptons invariant mass coming from a fermionic leptoquark, $J_{3}$, decaying into a bottom quark and $U$, i.e., $J_{3} \rightarrow b U^{++} \rightarrow b l^{+} l^{+}$, as showed in Fig. 7. This would be easy to observe within the luminosity level already reached by the LHC [58].
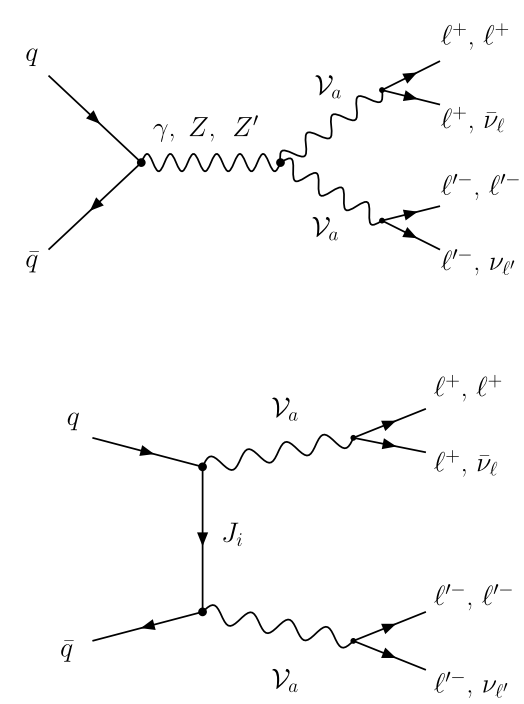

Fig. 6 Pair production and decay of new vector bosons. In the diagrams $\mathcal{V}_{a}$ represents $V^{ \pm}$or $U^{ \pm \pm}$

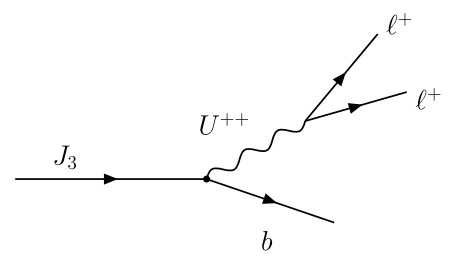

Fig. 7 Decay of a leptoquark $J_{3}$ into a b-jet plus two leptons from $U$

Also, by considering the future linear collider, clear signals will be also detected for example in the production of a same sign muon pair through the process $e^{-} e^{-}$or that of four leptons in $e^{-} e^{+}$collisions $[59,60]$.

\section{Conclusions and outlook}

The goal of discovering the Higgs boson has finally been achieved at the LHC and corroborated by evidence at the Tevatron. In fact, telling the resonance is a Higgs boson is nothing but a pretty good guess in this moment. The next logical effort, both experimental and theoretical, is to study the new particle's properties in order to confirm or not its role in the electroweak symmetry breaking.

Some beyond SM models are able to explain the current state of affairs concerning the branching ratios of the hypothesized Higgs, however, it is not generically easy to adjust the couplings of the Higgs to SM particles in order to fit the current data. One of the most economical alternatives to this scenario would be to keep all SM couplings untouched and add new states to which the Higgs could decay to, decreasing the branching ratios globally at the same time these new states enhance the 1-loop coupling to photons. 
A few models realize this situation in a more natural fashion including the classes of 331 models considered in this work, in particular, the versions presenting a Cold Dark Matter particle. In these realizations, the Higgs boson may decay to CDM particles and become invisible to the detectors while the couplings to the rest of the spectrum would look like SM.

In this work we show that the minimal 331 model, and versions presenting a dark matter candidate, can fit the current data within the experimental errors. We performed a $\chi^{2}$ analysis using the publicly available data (with unknown correlations) and found that the proposed models with charged vector masses of $212.5 \mathrm{GeV}$ and a branching ratio to invisible states of $17 \%$ are the preferred parameters from the fitting procedure. Furthermore, given the large errors, larger masses and smaller branching ratios to invisible states cannot be excluded. The preferred signal strength to photons from the fitting procedure is $\mu_{\gamma \gamma}=1.57$, which agrees well with the LHC data. If no DM is present in Higgs decays, our analysis finds $\mu_{\gamma \gamma}=1.59$ and $m_{V}=262.5 \mathrm{GeV}$ as the best fitted mass.

A general prediction of 331 models with a Cold Dark Matter candidate is to globally decrease the branching ratios to SM particles. Given the observation of deficits in many experimental data, and allowing an invisible decay channel, as, in fact, is predicted by supersymmetric 331 models and minimal 331 models embedded in larger groups, as the 341 , our $\chi^{2}$ analysis shows that the best global signal strength to all SM particles, but the photon, fitted to the data is $\mu_{s s}=0.83$. Comparing this to the CMS global signal strength $\sigma / \sigma_{\mathrm{SM}}=0.80 \pm 0.22$, we may say that the 331 explanation to the Higgs branching ratios and the signal strength in diphoton channel is robust within the current experimental errors.

Whatever the direction the experimental picture evolves, these models are able to describe a scenario with either decreased branching ratios into $Z, W$ and $b, \tau$ pairs or SM branching fractions, and either a photophilic or a SM Higgs concerning the branching to photons. Nevertheless, the charged vector bosons masses preferred by the data suggest that a direct search for new gauge bosons from the models considered here is well within the reach of the LHC. We also point out that if it could be possible to compare the yields of the associated process $p p \rightarrow h Z(W) \rightarrow \gamma \gamma Z(W)$ with the SM prediction this would help to distinguish between the 331 models and other models that globally change the branching ratios of the Higgs into SM particles. All of this could be combined with signals coming from peculiar processes that we have mentioned above involving $U$ and $V$ in order to probe the extended electroweak models we deal with here.

Acknowledgements This work was supported by Conselho Nacional de Pesquisa e Desenvolvimento Científico-CNPq, Coorde- nação de Aperfeiçoamento Pessoal de Nível Superior-CAPES, and Fundação de Amparo à Pesquisa do Estado de São Paulo-FAPESP.

\section{References}

1. J. Incandela, CMS talk at latest update in the search for the Higgs boson at CERN, July 4, 2012 [Slides]

2. F. Gianotti, ATLAS talk at latest update in the search for the Higgs boson at CERN, July 4, 2012 [Slides]

3. F. Englert, R. Brout, Phys. Rev. Lett. 13, 321 (1964)

4. P.W. Higgs, Phys. Rev. Lett. 13, 508 (1964)

5. G.S. Guralnik, C.R. Hagen, T.W.B. Kibble, Phys. Rev. Lett. 13, 585 (1964)

6. E. James, W. Fisher. Tevatron Higgs seminar, July 2, 2012 [Slides]

7. A. Alves, E.R. Barreto, A.G. Dias, F.S. Queiroz, C.A. Pires, P.S.R. da Silva, Phys. Rev. D 84, 115004 (2011). arXiv:1109.0238 [hep$\mathrm{ph}]$

8. F. Pisano, V. Pleitez, Phys. Rev. D 46, 410 (1992). hep-ph/ 9206242

9. P.H. Frampton, Phys. Rev. Lett. 69, 2889 (1992)

10. V. Pleitez, M.D. Tonasse, Phys. Rev. D 48, 2353 (1993). hep-ph/ 9301232

11. M. Carena, I. Low, C.E.M. Wagner, arXiv:1206.1082 [hep-ph]

12. J. Gunion, X. Jaing, S. Kram, arXiv:1207.1545 [hep-ph]

13. A. Arbeya, M. Battaglia, A. Djouadi, F. Mahmoudi, arXiv: 1207.1348 [hep-ph]

14. R. Benbrik, M.G. Bock, S. Heinemeyer, O. Stal, G. Weiglein, L. Zeune, arXiv:1207.1096 [hep-ph]

15. S. Chang, C.A. Newby, N. Raj, C. Wanotayaroj, arXiv:1207.0493 [hep-ph]

16. V. Barger, M. Ishida, W.-Y. Keung, arXiv:1207.0779 [hep-ph]

17. B. Bellazzini, C. Petersson, R. Torre, arXiv:1207.0803 [hep-ph]

18. K. Cheung, T.-C. Yuan, Phys. Rev. Lett. 108, 141602 (2012). arXiv:1112.4146 [hep-ph]

19. H.S. Cheon, S.K. Kang, arXiv:1207.1083 [hep-ph]

20. M. Montull, F. Riva, arXiv:1207.1716 [hep-ph]

21. J. Ellis, T. You, arXiv:1207.1693 [hep-ph]

22. T. Corbett, O.J.P. Eboli, J. Gonzalez-Fraile, M.C. GonzalezGarcia, arXiv:1207.1344 [hep-ph]

23. J.R. Espinosa, C. Grojean, M. Muhlleitner, M. Trott, arXiv: 1207.1717 [hep-ph]

24. P.P. Giardino, K. Kannike, M. Raidal, A. Strumia, arXiv: 1207.1347 [hep-ph]

25. D. Carmi, A. Falkowski, E. Kuflik, T. Volansky, J. Zupan, arXiv:1207.1718 [hep-ph]

26. J.D.R. Alvares, C.A. de S. Pires, F.S. Queiroz, D. Restrepo, P.S. Rodrigues da Silva, arXiv:1206.5779 [hep-ph]

27. J. Ellis, M.K. Gaillard, D. Nanopoulos, Nucl. Phys. B 106, 292 (1976)

28. A.I. Vainshtein, M.B. Voloshin, V.I. Zakharov, M.A. Shifman, Sov. J. Nucl. Phys. 30, 711 (1979)

29. L.B. Okun, Leptons and Quarks (North-Holland, Amsterdam, 1982)

30. J.F. Gunion, H.E. Haber, G. Kane, S. Dawson, The Higgs Hunter's Guide (Perseus Books, Cambridge, 1990)

31. M.B. Voloshin, Sov. J. Nucl. Phys. 48, 512 (1988)

32. P.R.D. Pinheiro, C.A. de S. Pires, P.S. Rodrigues da Silva, in preparation

33. T.V. Duong, E. Ma, Phys. Lett. B 316, 307 (1993). hep-ph/ 9306264

34. J.C. Montero, V. Pleitez, M.C. Rodriguez, Phys. Rev. D 65, 035006 (2002). hep-ph/0012178

35. S.P. Martin, hep-ph/9709356 
36. J.G. Ferreira Jr., C.A. de S. Pires, P.S. Rodrigues da Silva, A. Sampieri, in preparation

37. A. Djouadi, J. Kalinowski, M. Spira, Comput. Phys. Commun. 108, 56 (1998). hep-ph/9704448

38. G. Aad et al. (ATLAS Collaboration), arXiv:1207.0319 [hep-ex]

39. S. Chatrchyan et al. (CMS Collaboration), Phys. Lett. B 710, 26 (2012). arXiv:1202.1488 [hep-ex]

40. Tevatron New Physics Higgs Working Group and CDF and D0 Collaborations, arXiv:1207.0449 [hep-ex]

41. G. Cowan, Statistical Data Analysis (Oxford University Press, Oxford, 1998)

42. K. Sasaki, Phys. Lett. B 308, 297 (1993)

43. P.H. Frampton, M. Harada, Phys. Rev. D 58, 095013 (1998). hep-ph/9711448

44. H.N. Long, T. Inami, Phys. Rev. D 61, 075002 (2000). hep-ph/9902475

45. A.G. Dias, J.C. Montero, V. Pleitez, Phys. Lett. B 637, 85 (2006). hep-ph/0511084

46. J. Beringer et al. (PDG Collaboration), Phys. Rev. D 86, 010001 (2012). http://pdg.lbl.gov

47. L. Willmann et al., Phys. Rev. Lett. 82, 49 (1999). hep-ex/ 9807011
48. M.B. Tully, G.C. Joshi, Phys. Lett. B 466, 333 (1993). hep-ph/9905552

49. E.M. Gregores, A. Gusso, S.F. Novaes, Phys. Rev. D 64, 015004 (2001). hep-ph/0101048

50. V. Pleitez, Phys. Rev. D 61, 057903 (2000). hep-ph/9905406

51. P.H. Frampton, D. Ng, Phys. Rev. D 45, 4240 (1992)

52. F. Cuypers, S. Davidson, Eur. Phys. J. C 2, 503 (1998)

53. B. Dion, T. Grégoire, D. London, L. Marleau, H. Nadeau, Phys. Rev. D 59, 075006 (1999). hep-ph/9810534

54. E. Ramirez Barreto, Y.A. Coutinho, J.S. Borges, Nucl. Phys. B 810, 210 (2009). arXiv:0811.0846

55. E. Ramirez Barreto, Y.A. Coutinho, J.S. Borges, Phys. Rev. D 83, 075001 (2011). arXiv:1103.1267

56. B. Meirose, A.A. Nepomuceno, Phys. Rev. D 84, 055002 (2011). arXiv:1105.6299 [hep-ph]

57. E. Ramirez Barreto, Y.A. Coutinho, J.S. Borges, work in progress

58. A. Alves, E.R. Barreto, A.G. Dias, Phys. Rev. D 86, 055025 (2012). arXiv:1203.2342 [hep-ph]

59. P.H. Frampton, A. Rasin, Phys. Lett. B 482, 129 (2000). hep-ph/0002135

60. E. Ramirez Barreto, Y.A. Coutinho, J.S. Borges, Phys. Lett. B 632, 675 (2006). hep-ph/0509355 